\title{
Evaluation of the safety of MRI scans in patients undergoing dual-chamber pacemaker implantation
}

\author{
MINCHAO XIONG ${ }^{*}$, NIAN ZHAO ${ }^{2 *}$, YAN QIN $^{2}$, PENG MIN $^{2}$, HONG PENG $^{2}$, \\ YONG ZHANG $^{2}$ and BIN FAN $^{3}$ \\ ${ }^{1}$ Department of Radiology, Ezhou Central Hospital, Ezhou, Hubei 436000; \\ ${ }^{2}$ Medical Imaging Center, Dongfeng Hospital of Hubei Medical College, Shiyan, Hubei 442008; \\ ${ }^{3}$ Department of Radiology, Central Hospital of Huanggang, Huanggang, Hubei 438000, P.R. China
}

Received June 22, 2017; Accepted October 6, 2017

DOI: $10.3892 / \mathrm{etm} .2018 .6392$

\begin{abstract}
The safety of magnetic resonance imaging (MRI) scans in patients undergoing dual-chamber pacemaker (Medtronic's EnRhythm MRI SureScan IPG (implantable pulse generator) and CapSureFix MRI pacing electrodes) implantation were evaluated. A total of 86 patients undergoing this pacemaker implantation who were admitted to Huanggang Hospital from June 2006 to March 2017 were continuously selected. On average 6.8 \pm 2.3 months after the implantation, the first MRI scan was conducted. The mean scan time was $2.2 \pm 0.9$ and mean duration was $45.6 \pm 12.3 \mathrm{~min}$; mean follow-up visit period was $40.5 \pm 15.6$ months, and after follow-up, all the abnormal symptoms of patients and pacemaker abnormalities after scans were recorded. Causes of receiving MRI scans included neurological diseases $(27 \%)$, spinal diseases $(14 \%)$, cancers $(26 \%)$, joint injuries $(25 \%)$ and visceral systems $(8 \%)$. A total of 12 cases $(14.0 \%)$ showed significant discomfort symptoms, 10 cases (11.6\%) showed pacemaker abnormalities, and the incidence rate was $25.6 \%$. Multivariate logistic regression analysis showed that the basic types of cardiovascular diseases, dependence on pacemakers, duration of education, pacing threshold, impedance and MRI scan time were related to the occurrence of adverse scan results $(\mathrm{p}<0.05)$. In conclusion, MRI scans show that MRI-compatible dual-chamber pacemaker was relatively safe.
\end{abstract}

\section{Introduction}

Permanent pacemaker implantation has become an important treatment for a variety of organic arrhythmias, but about

Correspondence to: Dr Bin Fan, Department of Radiology, Central Hospital of Huanggang, 11 Kaopeng Street, Huangzhou, Huanggang, Hubei 438000, P.R. China

E-mail: gk0004440@163.com

*Contributed equally

Key words: EnRhythm MRI SureScan pacemaker, MRI, safety
$50-75 \%$ of patients are required to receive nuclear magnetic resonance imaging (MRI) scans after implantation (1). MRI does not involve radiation and has multi-directional imaging as well as excellent contrast resolution of soft tissues, so it can provide relatively more comprehensive information about tissue perfusion, function and metabolism. Besides, it can also clearly show the heart, blood vessels and body cavity without contrast agents, and its cardiac function evaluation also has a higher application value (2). Components and materials of conventional cardiac pacemakers contain ferromagnetic substance, so under the action of high field strength of static fields, the pulse generator and pacing electrodes can be turned or shifted (3); radio frequency fields interfere with magnetic components to partly or fully disable them in ways such as closing reeds, changing the frequency of pacemakers, resetting programs or inhibiting pace-making (4). Thermal energy generated at the junction of the top of electrodes and myocardial tissues injures the myocardia, thus contributing to the formation of scars or perforations, and it also affects the perception and program-controlled functions of the device, thus inducing arrhythmia (5). On the area around $15 \mathrm{~cm}$ of the pulse generator, there appear heavy artifacts (6). In 2008, the first EnRhythm MRI SureScan IPG pacemaker and CapSureFix MRI pacing electrode (Type: 5086MRI) of Medtronic were approved by the European Conformity (CE) and U.S. Food and Drug Administration (FDA) and clinical safety studies have been conducted $(7,8)$. Up to now, the largest sample study was conducted for 723 patients from 66 heart centers in the United States. About $13 \%$ of patients were followed up for 1 year and received MRI scans for neurological diseases (33\%), spinal diseases $(16 \%)$, cancers $(11 \%)$, joint injuries $(11 \%)$ and other causes $(29 \%)$, and $47 \%$ of patients did not receive MRI and CT scans before pacemaker implantation. There appeared no serious symptoms and pacemaker dysfunctions in any of the patients (9). The aim of this study was to evaluate the safety of MRI scans in pacemaker implantation and present precautions.

\section{Patients and methods}

Patient information. A total of 86 patients undergoing this pacemaker implantation who were admitted to Huanggang 
Hospital (Huanggang, China) from June 2006 to March 2017 were continuously selected. Among them, there were 45 males and 41 females aged from 45 to 78 years with the mean age of $56.5 \pm 14.3$ years. The duration of operation was $46-82 \mathrm{~min}$ with the mean value of $65.3 \pm 19.8 \mathrm{~min}$. The basic types of cardiovascular diseases: Severe slow rhythm (49 cases), persistent atrial fibrillation after radiofrequency ablation (12 cases), severe ventricular rhythm (15 cases) and severe coronary heart disease or heart failure with arrhythmia (10 cases). There were 35 cases of pacemaker-dependent type and 51 cases of pacemaker-independent type. Pacing modes: AAI (A, atrial paced; A, atrial sensed; I, pacing is inhibited if beat is sensed) (38 cases), VVI (V, ventricle paced; V, ventricle sensed; I, pacing is inhibited if beat is sensed) (25 cases) and DDR (D, both atrial and ventricle paced; D, both atrial and ventricle sensed; R, rate responsive) (23 cases); there were 26 cases accompanied with hypertension, 12 cases with diabetes and 20 cases with stroke; and there were 30 cases who had received MRI scans before the pacemaker implantation and 56 cases with no such history. Education duration was 9-15 years with the average value of $11.2 \pm 3.5$ years. The study was approved by the Ethics Committee of Ezhou Central Hospital (Hubei, China) and patients signed written informed consents.

Study methods. The study was completed by the same team for MRI scans and nursing. They informed patients risks in scans and signed safety questionnaires. The whole process was accompanied by specialist physicians. Before scans, the pacemaker was adjusted to the MRI-compatible mode [AOO (A, atrial paced; O, none sensed; O, none-rate responsive), VOO (V, ventricle paced; $\mathrm{O}$, none sensed; $\mathrm{O}$, none-rate responsive), DOO (D, both atrial and ventricle paced; $\mathrm{O}$, none sensed; O, none-rate responsive) and $\mathrm{OFF}]$ with the pacing threshold $\leq 2.0 \mathrm{~V} / 0.4 \mathrm{~m} / \mathrm{sec}$. The low resistance value was 200-1,500 $\Omega$ and the high resistance value was 30-100 $\Omega$. First aid equipment and drugs were prepared. The low field strength was $(<1.5 \mathrm{~T})$, and the gradient switching rate was $\leq 200 \mathrm{~T} / \mathrm{m} / \mathrm{sec}$. Synchronized electrocardiogram (ECG) monitoring was conducted. Body radiation absorption rate was $\leq 2.0 \mathrm{~W} / \mathrm{kg}$; the radiation rate of the head was $\leq 3.2 \mathrm{~W} / \mathrm{kg}$; the duration of scan in pacemaker implantation for the first time was $\geq 6$ weeks. The type of the MRI scanner was Siemens Sonata superconductive 1.5T, and we selected different scan sequences according to different inspection sites.

Observation indexes. Follow-up duration was 2-80 months with the mean value of $40.5 \pm 15.6$ months. Mean time of first MRI scan, scan times and mean scan duration after pacemaker implantation were recorded. Causes were examined, and according to all the symptoms and abnormalities of pacemakers appearing in patients after the examination, patients were divided into the discomfort group and the normal group, and possible causes of discomfort were analyzed.

Statistical analysis. SPSS 20.0 (IBM Corp., Armonk, NY, USA) was used for statistical analysis. Measurement data were represented as mean \pm standard deviation. Risk factors affecting MRI scans were analyzed by multivariate logistic regression analysis and screened by using progressive

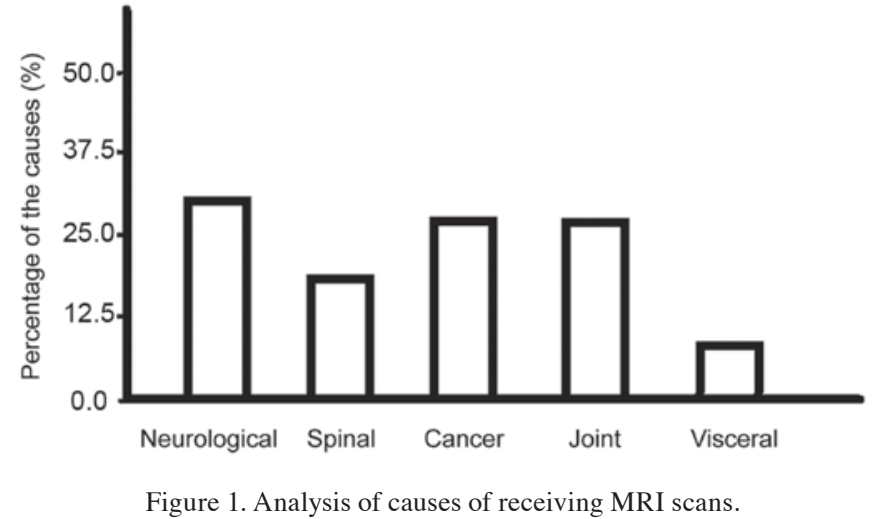

retrac-tion method. $\mathrm{P}<0.05$ was considered to indicate a statistically significant difference.

\section{Results}

Mean time of the first MRI scan, scan times and mean scan duration. After implantation (2-15 months), the first MRI scan was conducted with the mean time of $6.8 \pm 2.3$ months; scan times was 1-5 times with the average value of $2.2 \pm 0.9$ times; scan duration was 35-68 min with the average value of $45.6 \pm 12.3 \mathrm{~min}$.

Causes. The main causes include neurological diseases (such as stroke, neurodegenerative diseases, spinal cord lesions and craniocerebral trauma), spinal diseases (such as disc herniation and traumatic fracture), cancers (such as bone tumors, brain tumors and breast tumors), joint injuries (such as femoral head necrosis, knee cruciate ligament injury and hip dislocation) and visceral system scans (hepatobiliary pancreas, prostate, ovary and uterus) (Fig. 1).

Discomfort symptoms and pacemaker abnormalities. There appeared discomfort symptoms in 12 patients among 86 patients, such as excessive anxiety, significantly higher blood pressure (10\% higher than the base value), significantly faster heart rate (10\% faster than the base value); there appeared pacemaker abnormalities in a total of 10 patients, such as increased impedance $(20 \%$ greater than the base value), abnormalities in perception or pacemakers (appeared pace-making and self-rhythm), disorders in perception or pacemakers (severe heart rate overrun or arrest), hypotension or blackness, no syncope and disturbance of consciousness. Safe scans could be achieved by psychological counseling, debugging pacemaker parameters, changing MRI scan sequences and other methods without pacemaker or wire shifting or other changes.

Risk factors affecting MRI scans. Data such as patient baseline data as well as MRI scan and pacemaker para-meters were included in the multivariate logistic regression analysis model. As independent variables, appearances of discomfort symptoms and pacemaker abnormalities were selected as dependent variables for screening. The final results showed that the basic types of cardiovascular diseases, dependence on pacemakers, duration of education, pacemaker threshold, impedance and 
Table I. Analysis of risk factors affecting MRI scans.

\begin{tabular}{lccccc}
\hline Factors & $\beta$ & Wald & P-value & OR & $95 \%$ CI \\
\hline Basic types of cardiovascular diseases & 0.123 & 5.236 & 0.018 & 1.625 & $1.128-2.659$ \\
Dependence on pacemakers & 0.285 & 4.625 & 0.029 & 1.147 & $1.007-3.065$ \\
Duration of education & -0.547 & 4.121 & 0.033 & 0.522 & $0.147-0.863$ \\
Pacemaker threshold & 0.352 & 4.554 & 0.031 & 1.085 & $1.021-2.659$ \\
Impedance & 0.427 & 4.329 & 0.035 & 1.072 & $1.011-2.954$ \\
MRI scan time & 0.441 & 4.859 & 0.025 & 1.326 & $1.124-2.836$ \\
\hline
\end{tabular}

MRI scan time were correlated with the occurrence of adverse outcomes $(\mathrm{p}<0.05)$ (Table I).

\section{Discussion}

The pacemaker has small volume, light weight and high magnetization resistance, good magnetic field stability and other advantages (10). Replacing pacemaker switches with Hall sensors increased magnetic field stability; replacing traditional ferromagnetic wires with steel fiber wires reduced wire impedance; shield protection for internal power supply circuits reduced the content of pacemaker ferromagnetic materials; the mode exclusive for MRI was started during the scan, and resumed after the scan. Under the high field strength (3T and $8 \mathrm{~T})$ and high specific absorption rate (SAR) $(3.90 \mathrm{~W} / \mathrm{kg})$, the scan was performed for a long time $(210 \mathrm{~min})$, and the results showed that the image quality was not reduced and there appeared no obvious discomfort in patients and no obvious dysfunction in the pacemaker (11).

The study included relatively more subjects, so the basic types of cardiovascular diseases were complex, including pacemaker-dependent and pacemaker-independent types and pacing modes were different. Duration of the first MRI scan was 2-15 months, and the perception, pacing function and electrode impedance of the pacemaker tended to reach a steady state for 1 to 2 weeks after operation, which needed to be programmed to be adjusted to the optimal state (12). Sequences needed to be scanned were different for different parts undergoing MRI scans, thus posing different levels of influence on the pacemaker working state, which has not yet been specifically analyzed. A total of 12 cases $(14.0 \%)$ showed significant discomfort symptoms, 10 cases $(11.6 \%)$ showed pacemaker abnormalities, and the incidence rate was $25.6 \%$. The study distinguished between patient discomfort and pacemaker abnormality, and the results showed that patient discomfort was often associated with poor communication and great psychological fluctuation, while pacemaker abnormality was more likely to be associated with MRI interference (13). For patients with neurological disorders, tumors, and osteoarthritis, it was advisable to select an MRI-compatible pacemaker, and for those who did not need MRI scan for the time being, using MRI-compatible pacing electrodes in patients in advance could retain the right to receive MRI scans in the future (8). Data and results of present studies are still limited to the scan under the $1.5 \mathrm{~T}$ or less field strength, and there still exists no large sample data about that under 3.0T field strength (14). Therefore, clinical selection of intended population still needs to be cautious.
Through screening, the study further showed that the basic types of cardiovascular diseases, dependence on pacemakers, duration of education, pacing threshold, impedance and MRI scan time were related to the occurrence of adverse outcomes. Patients with severe ventricular arrhythmia (15 cases) and severe coronary heart disease or heart failure with arrhythmia were more likely to be examined with adverse events after postoperative radiofrequency ablation of persistent atrial fibrillation among patients with the basic types of cardiovascular diseases. Possible causes might be poor cardiac rhythm and dependence on pacemaker $(15,16)$. Patients dependent on pacemakers tended to have high pacing thresholds and relatively higher impedance (17). The longer the duration of education was, the more clearly the pacemaker maintenance and MRI scan precautions would be, which could reduce the incidence rate of adverse events. And the longer the duration of MRI scans was, the higher the probability of resulted uncontrollable pacing abnormalities would be $(18,19)$. This study provided an important reference for guiding clinical MRI and pre-scan preparation.

\section{Acknowledgements}

Not applicable.

\section{Funding}

No funding was received.

\section{Availability of data and materials}

The datasets used and/or analyzed during the present study are available from the corresponding author on reasonable request.

\section{Authors' contributions}

MX, NZ and BF conceived and designed the study. MX, YQ and PM were responsible for the collection and analysis of the patient data. NZ, HP and YZ interpreted the data and drafted the manuscript. BF revised the manuscript critically for important intellectual content. All authors read and approved the final manuscript.

\section{Ethics approval and consent to participate}

The study was approved by the Ethics Committee of Ezhou Central Hospital (Hubei, China) and patients signed written informed consents. 


\section{Patient consent for publication}

Not applicable.

\section{Competing interests}

The authors declare that they have no competing interests.

\section{References}

1. Jung W, Zvereva V, Hajredini B and Jäckle S: Safe magnetic resonance image scanning of the pacemaker patient: Current technologies and future directions. Europace 14: 631-637, 2012.

2. Nordbeck P, Ertl G and Ritter O: Magnetic resonance imaging safety in pacemaker and implantable cardioverter defibrillator patients: How far have we come? Eur Heart J 36: 1505-1511, 2015

3. Li Y, Han Z, Sun Y, Li A, Zhang W, Li A and Liu S: Endoscopic polypectomy for pacemaker patients: Is it safe? ANZ J Surg 85: 834-837, 2015.

4. Mesubi O, Ahmad G, Jeudy J, Jimenez A, Kuk R, Saliaris A, See V, Shorofsky S and Dickfeld T: Impact of ICD artifact burden on late gadolinium enhancement cardiac MR imaging in patients undergoing ventricular tachycardia ablation. Pacing Clin Electrophysiol 37: 1274-1283, 2014

5. Klein-Wiele O, Garmer M, Urbien R, Busch M, Kara K, Mateiescu S, Grönemeyer D, Schulte-Hermes M, Garbrecht M and Hailer B: Feasibility and safety of adenosine cardiovascular magnetic resonance in patients with MR conditional pacemaker systems at 1.5 Tesla. J Cardiovasc Magn Reson 17: 112, 2015.

6. Levine GN, Gomes AS, Arai AE, Bluemke DA, Flamm SD, Kanal E, Manning WJ, Martin ET, Smith JM, Wilke N, et al; American Heart Association Committee on Diagnostic and Interventional Cardiac Catheterization; American Heart Association Council on Clinical Cardiology; American Heart Association Council on Cardiovascular Radiology and Intervention: Safety of magnetic resonance imaging in patients with cardiovascular devices: an American Heart Association scientific statement from the Committee on Diagnostic and Interventional Cardiac Catheterization, Council on Clinical Cardiology, and the Council on Cardiovascular Radiology and Intervention: endorsed by the American College of Cardiology Foundation, the North American Society for Cardiac Imaging, and the Society for Cardiovascular Magnetic Resonance. Circulation 116: 2878-2891, 2007.

7. Ferreira AM, Costa F, Tralhão A, Marques H, Cardim N and Adragão P: MRI-conditional pacemakers: Current perspectives. Med Devices (Auckl) 7: 115-124, 2014.

8. Shinbane JS, Colletti PM and Shellock FG: Magnetic resonance imaging in patients with cardiac pacemakers: Era of 'MR Conditional' designs. J Cardiovasc Magn Reson 13: 63, 2011
9. Wollmann CG, Thudt K, Kaiser B, Salomonowitz E, Mayr H and Globits S: Safe performance of magnetic resonance of the heart in patients with magnetic resonance conditional pacemaker systems: the safety issue of the ESTIMATE study. J Cardiovasc Magn Reson 16: 30, 2014.

10. Sutton R, Kanal E, Wilkoff BL, Bello D, Luechinger R, Jenniskens I, Hull M and Sommer T: Safety of magnetic resonance imaging of patients with a new Medtronic EnRhythm MRI SureScan pacing system: Clinical study design. Trials 9: 68, 2008.

11. Nazarian S, Hansford R, Roguin A, Goldsher D, Zviman MM, Lardo AC, Caffo BS, Frick KD, Kraut MA, Kamel IR, et al: A prospective evaluation of a protocol for magnetic resonance imaging of patients with implanted cardiac devices. Ann Intern Med 155: 415-424, 2011.

12. Roguin A, Schwitter J, Vahlhaus C, Lombardi M, Brugada J, Vardas P, Auricchio A, Priori S and Sommer T: Magnetic resonance imaging in individuals with cardiovascular implantable electronic devices. Europace 10: 336-346, 2008.

13. Nazarian S, Beinart R and Halperin HR: Magnetic resonance imaging and implantable devices. Circ Arrhythm Electrophysiol 6: 419-428, 2013.

14. Gimbel JR: Unexpected pacing inhibition upon exposure to the 3T static magnetic field prior to imaging acquisition: What is the mechanism? Heart Rhythm 8: 944-945, 2011

15. Beinart R and Nazarian S: Effects of external electrical and magnetic fields on pacemakers and defibrillators: From engineering principles to clinical practice. Circulation 128: 2799-2809, 2013

16. Tandri H, Zviman MM, Wedan SR, Lloyd T, Berger RD and Halperin H: Determinants of gradient field-induced current in a pacemaker lead system in a magnetic resonance imaging environment. Heart Rhythm 5: 462-468, 2008.

17. Calcagnini G, Triventi M, Censi F, Mattei E, Bartolini P, Kainz W and Bassen HI: In vitro investigation of pacemaker lead heating induced by magnetic resonance imaging: Role of implant geometry. J Magn Reson Imaging 28: 879-886, 2008.

18. Mollerus M, Albin G, Lipinski M and Lucca J: Cardiac biomarkers in patients with permanent pacemakers and implantable cardioverter-defibrillators undergoing an MRI scan. Pacing Clin Electrophysiol 31: 1241-1245, 2008.

19. Nordbeck P, Weiss I, Ehses P, Ritter O, Warmuth M, Fidler F, Herold V, Jakob PM, Ladd ME, Quick HH, et al: Measuring RF-induced currents inside implants: Impact of device configuration on MRI safety of cardiac pacemaker leads. Magn Reson Med 61: 570-578, 2009.

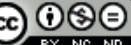

This work is licensed under a Creative Commons Attribution-NonCommercial-NoDerivatives 4.0 International (CC BY-NC-ND 4.0) License. 